\title{
Treatment outcomes and factors affecting treatment outcomes of new patients with tuberculosis in Busan, South Korea: a retrospective study of a citywide registry, 2014-2015
}

Jeongha Mok ${ }^{1,2,3}$, Daeseong An ${ }^{4}$, Seoungjin $\mathrm{Kim}^{3}$, Miyoung Lee ${ }^{3}$, Changhoon Kim ${ }^{2,3,5}$ and Hyunjin Son ${ }^{3^{*}}$ (D)

\begin{abstract}
Background: This study investigated the treatment outcomes, and factors affecting the outcomes, of new tuberculosis (TB) patients in Busan, South Korea.

Methods: We retrospectively analysed the citywide TB registry data (collected for the Korean National TB Surveillance System) of new TB patients registered in Busan from January 2014 to December 2015.

Results: A total of 4732 patients were included in this study (mean age, $52.5 \pm 19.9$ years; $58.4 \%$ male). The overall treatment success rate was $83.9 \%$ (cured, 20.2\%; completed, $63.7 \%$ ); $8.0 \%$ of patients died, and 3.6\% were lost to follow-up. In multivariate analyses, a higher rate of loss to follow-up was associated with foreign nationality, registered as TBpositive at least twice, and being in Q4 (fourth quintile) or Q5 (fifth quintile) of the regional deprivation index. Conversely, a lower rate of loss to follow-up was associated with female gender, smear-positive for pulmonary TB (PTB), and the treatment outcome being reported by a public health centre. Higher mortality was associated with old age ( $\geq 75$ years), smear-positive PTB, treatment outcome being reported by the hospital, and being registered as TB-positive twice. Lower mortality was associated with female gender, treatment outcome being reported by a public health centre or clinic, and Q5 of the regional deprivation index.
\end{abstract}

Conclusions: Treatment outcomes of new TB patients were sub-optimal in Busan. TB control programs should maintain close monitoring and provide greater socioeconomic support to patients at high risk of poor treatment outcomes.

Keywords: Outcome, South Korea, Tuberculosis

\section{Background}

Despite efforts to eliminate tuberculosis (TB), the incidence and mortality of TB remain high worldwide. In 2016 alone, there were 10.4 million new TB cases globally, and 1.7 million people died from the disease [1]. South Korea is not free from TB, despite having abundant medical resources. In 2017, a total of 36,044 patients in South Korea infected with TB $(70.4 / 100,000$

\footnotetext{
* Correspondence: hjson78@gmail.com

${ }^{3}$ Busan Center for Infectious Disease Control and Prevention, Pusan National University Hospital, 179 Gudeok-ro, Seo-gu, Busan 49241, South Korea Full list of author information is available at the end of the article
}

population) were listed on the Korean National TB Surveillance System (NTSS), and $78 \%$ of them $(28,161$ patients; 55.0/100,000 population) were new patients [2]. A total of 2186 patients died from TB in South Korea in 2016 [2]. South Korea has the highest TB incidence and mortality rate of all member countries of the Organization for Economic Co-operation and Development [3], and TB remains a serious public health problem in South Korea.

The "End TB Strategy" of the World Health Organization (WHO) has set a target to increase the TB treatment success rate to $90 \%$ by 2025 , and to reduce $\mathrm{TB}$ 
deaths by $95 \%$ (compared to 2015) by 2035 , to end the global TB epidemic [4]. To facilitate these goals and evaluate the effectiveness of the strategy, it is essential to accurately record and report the treatment outcomes TB patients. Treatment outcomes, as measured by a standardised method, are key indicators of national TB program (NTP) effectiveness [5]. In addition to monitoring treatment outcomes, improving treatment success rates is important and depends on identifying vulnerable populations and the risk factors for poor treatment outcomes. This information is important for policymakers in terms of resource planning, prioritisation and distribution.

To achieve the WHO's TB targets, South Korea has made many changes to its NTP since 2011. The Korea Centers for Disease Control and Prevention (KCDC) outlined the "New 2020 Plan," which set a target to cut the TB incidence rate in half by $2020(<50 / 100,000$ population) [3]. Several programs were implemented to reach this goal, including expanded public-private mix (PPM) collaborations, reinforcement of outbreak investigations, contact investigations and treatment of latent TB infection, forced hospitalisation for non-adherent patients and those with multidrug-resistant (MDR) TB, and making all hospital visits for diagnosis and treatment of TB free-of-charge.

Despite these efforts to improve TB treatment in South Korea, there are limited data regarding recent treatment outcomes of South Korean TB patients and little is known about the effects of the 2011 policy changes. Factors affecting treatment outcomes of South Korean TB patients are not well understood. Most previous studies included only a small number of patients, were conducted in a single institution, or were conducted solely in the private sector [6-8]; therefore, the overall status of TB in South Korea is poorly understood. In the present study, we investigated treatment outcomes of new patients with TB in Busan, which is one of the largest and most TB-prevalent cities in South Korea, using a citywide registry. We also evaluated the factors affecting treatment outcomes of TB patients.

\section{Methods}

\section{Study design and population}

This retrospective cohort study was conducted in Busan, which is the second-largest city in South Korea, with a population of 3.5 million in 2015 (6.8\% of the total South Korean population). Notified TB cases in Busan made up $7.5 \%$ of all notified TB cases in South Korea. The incidence rates of total and new TB cases in Busan were 87.6 and $68.6 / 100,000$ population in 2015 , respectively [9]. The incidence rate of human immunodeficiency virus-positive TB was less than $1 / 100,000$ population in 2015 [10].
All TB patients registered in Busan from January 2014 to December 2015 were screened for inclusion. Among them, new TB patients were included in the analysis. Patients with rifampin-resistant (RR) or MDR-TB were excluded because the long duration of treatment required for these strains made it difficult to confirm treatment outcomes at the time of data collection. Patients with an unknown treatment history, and whose final diagnosis was changed to a disease other than $\mathrm{TB}$, were also excluded. A patient could be registered more than once if they had been transferred from one institution to another during treatment. In total, 1021 (21.6\%) of the patients in our cohort were registered at two or more different institutions. We regarded this patient as one case from the beginning of data extraction.

Almost all of the registered TB patients received treatment according to Korean guidelines for TB [11], i.e. a 6-month, self-administered regimen consisting of isoniazid, rifampin, ethambutol and pyrazinamide for 2 months, followed by isoniazid, rifampin, and/or ethambutol for 4 months. An alternative regimen (isoniazid, rifampin, and ethambutol for 9 months) was administered in cases of pyrazinamide intolerance.

The present study protocol was reviewed and approved by the Institutional Review Board of Pusan National University Hospital (IRB approval number: C-1805-001-066). The requirement for obtaining informed consent was waived by the Institutional Review Board of Pusan National University Hospital because this was a retrospective study of routinely collected monitoring data. The dataset was private and provided by the Department of Health and Hygiene of Busan City. Administrative permission for review and analysis of the patient's record was obtained from the Department of Health and Hygiene of Busan City. The data were de-identified prior to being analysed.

\section{Data source}

TB is a mandatorily notifiable disease in South Korea. All patients diagnosed with TB are registered with the Korean NTSS, which is a web-based registration system launched in 2000 by the KCDC. The citywide registry data used in the present study were a subset of this Korean NTSS data. The Korean NTSS includes the following information: identification number, age, sex, nationality, address, TB case classification (based on history of previous $\mathrm{TB}$ treatment), type of $\mathrm{TB}$ (pulmonary TB [PTB] or extrapulmonary TB [EPTB]), type of institution that registered the patient initially, type of institution that reported the final treatment outcome, results of acid fast bacilli (AFB) smear and culture, drug susceptibility test results, and treatment outcome.

A flowchart showing the process followed to establish the study cohort of new TB patients registered in Busan 
in 2014-2015 is presented in Fig. 1. The registry data of TB patients in Busan were downloaded from the Korean NTSS server in November 2016, so the treatment outcomes of our 2014-2015 study cohort included follow-up for at least 11 months after diagnosis. For patients who were registered as TB-positive more than once, we selected the latest treatment outcomes. However, if the latest treatment outcomes were "not evaluated," we examined the notified raw data of each patient and, if possible, selected treatment outcomes other than "not evaluated."

\section{Definitions}

All definitions used in the study were in accordance with WHO definitions [5]. Patients were classified into two groups according to their TB treatment history: new patients were those who had never been treated for TB or who had taken anti-TB drugs for $<1$ month, and previously treated patients were those who had received anti-TB drugs for $\geq 1$ month. Treatment outcomes were categorised as follows: cured (PTB patient with bacteriologically confirmed $\mathrm{TB}$ at the beginning of treatment who was smear- or culture-negative in the last month of treatment and on at least one previous occasion; treatment completed (TB patient who completed treatment without evidence of failure but with no record of being cured); treatment failed ( $\mathrm{TB}$ patient whose sputum smear or culture was positive at month 5 or later during treatment); died (TB patient who died for any reason before or during the course of treatment); lost to follow-up (TB patient who did not start treatment or whose treatment was interrupted for 2 consecutive months or

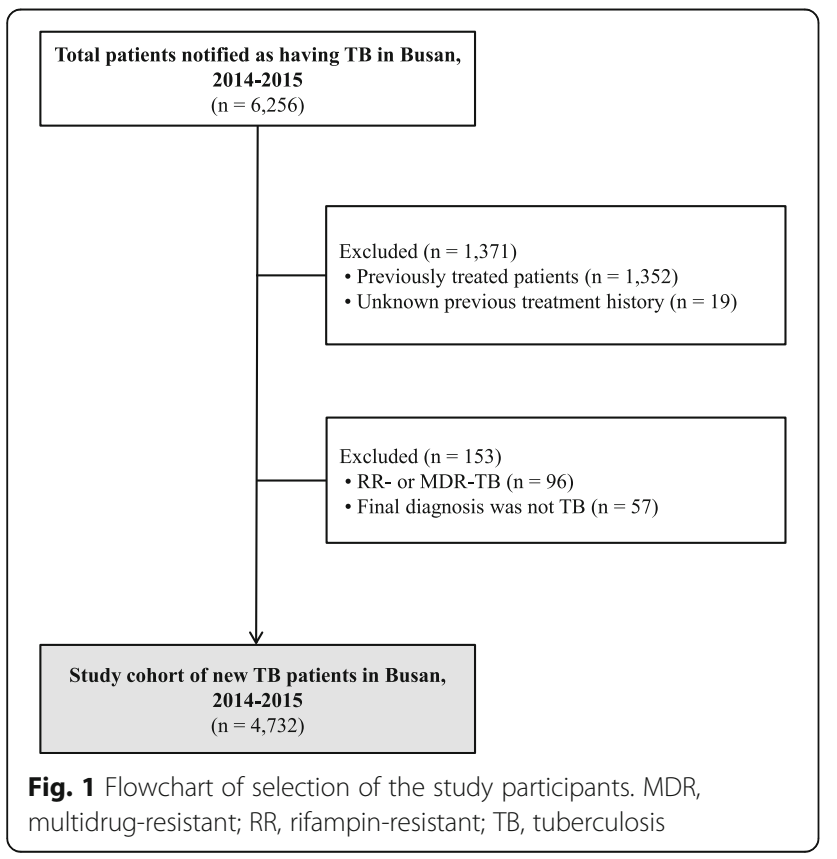

more); and not evaluated (TB patient to whom no treatment outcome was assigned, including cases that transferred to another institution). We defined treatment success as the sum of cured and treatment-completed cases. In 2015, the KCDC revised the definition of "cured" by eliminating the smear criterion. Thus, in the 2015 cohort, the treatment outcome "cured" was evaluated based only on culture results.

The Korean NTSS does not record socioeconomic data of patients. Therefore, we used a regional deprivation index. The regional deprivation index is used to estimate the socioeconomic status of an area (town-level by address in our study), and is regarded as an indirect indicator of the socioeconomic status of patients in a given area. The index is the sum of the following nine socioeconomic variables: house environment, educational level, social class of head-of-household and proportion of elderly people, single household, household without a car, non-apartment housing, female-headed household, and divorced or separated household. Higher index values indicate more deprivation, with Q5 (fifth quintile) corresponding to the most severe deprivation, and Q1 (first quintile) to the lowest level of deprivation [12].

\section{Statistical analyses}

Continuous variables are expressed as means \pm standard deviation and categorical variables as percentages. Continuous and categorical variables were compared using ANOVAs and chi-squared tests, respectively. To evaluate the factors affecting treatment outcomes, multivariate logistic regression was performed. Sensitivity analysis was also performed, in which all patients listed as "not evaluated" were assumed to be "lost to follow-up." All statistical analyses were performed using SAS software (ver. 9.4; SAS Institute Inc., Cary, NC, USA). Two-tailed $p$ values $<0.05$ were accepted as statistically significant.

\section{Results}

\section{Baseline characteristics}

A total of 6256 patients were screened during the study period. After application of the criteria outlined above, 4732 patients were included in the study cohort (Fig. 1). Baseline characteristics of the patients are summarised in Table 1. The mean age was $52.5 \pm 19.9$ years, and $58.4 \%$ were male; $1.6 \%(n=78)$ of total patients were of foreign nationality. РTB was the most common type of TB $(n=3771,79.7 \%)$, and $38.8 \%$ of the PTB patients showed positive smear results. Regarding the notifying institution, the private sector institutions notified over $90 \%$ of the study cohort $(n=4262)$. There was no significant difference in baseline characteristics between 2014 and 2015. 
Table 1 Baseline characteristics of the study cohort

\begin{tabular}{|c|c|}
\hline & $n=4732$ \\
\hline \multicolumn{2}{|l|}{ Year notified as having tuberculosis } \\
\hline 2014 & $2455(51.9)$ \\
\hline 2015 & $2277(48.1)$ \\
\hline \multicolumn{2}{|l|}{ Age, years } \\
\hline $0-9$ & $11(0.2)$ \\
\hline $10-19$ & $196(4.1)$ \\
\hline $20-44$ & $1451(30.7)$ \\
\hline $45-59$ & $1190(25.1)$ \\
\hline $60-74$ & $1117(23.6)$ \\
\hline$\geq 75$ & $767(16.2)$ \\
\hline \multicolumn{2}{|l|}{ Sex } \\
\hline Male & $2765(58.4)$ \\
\hline Female & $1967(41.6)$ \\
\hline \multicolumn{2}{|l|}{ Nationality } \\
\hline Korean & $4654(98.4)$ \\
\hline Foreign & $78(1.6)$ \\
\hline \multicolumn{2}{|l|}{ Type of tuberculosis } \\
\hline Pulmonary & $3771(79.7)$ \\
\hline Smear positive & $1464(30.9)$ \\
\hline Smear negative & $2307(48.8)$ \\
\hline Extrapulmonary & $961(20.3)$ \\
\hline \multicolumn{2}{|l|}{ Type of institution initially registering patient } \\
\hline Public health centre & $470(9.9)$ \\
\hline Private & $4262(90.1)$ \\
\hline PPM participating general hospital & $2534(53.6)$ \\
\hline PPM non-participating general hospital & $1026(21.7)$ \\
\hline Hospital & $478(10.1)$ \\
\hline Clinic & $224(4.7)$ \\
\hline \multicolumn{2}{|l|}{ Number of registrations as TB-positive } \\
\hline 1 & $3711(78.4)$ \\
\hline 2 & 849 (17.9) \\
\hline$\geq 3$ & $172(3.6)$ \\
\hline \multicolumn{2}{|l|}{ Regional deprivation index (quintile) ${ }^{a}$} \\
\hline Q1 & $1135(24.4)$ \\
\hline Q2 & $1237(26.6)$ \\
\hline Q3 & $1213(26.0)$ \\
\hline Q4 & $729(15.7)$ \\
\hline Q5 & $344(7.4)$ \\
\hline
\end{tabular}

PPM public-private mix, TB tuberculosis

Data are presented as numbers (\%)

${ }^{a} n=4658$ (variables of 74 patients were missing)

\section{Treatment outcomes}

Treatment outcomes are listed in Table 2. The overall treatment success rate was $83.9 \%$ (cured, 20.2\%; completed, $63.7 \%$ ) and 8.0 and $3.6 \%$ of patients died and were lost to follow-up, respectively. Treatment outcomes of "completed" and "not evaluated" were more common in 2015 than in 2014.

Table 3 shows the treatment outcomes according to patient characteristics. Higher treatment success rates were seen in patients who were younger (especially those aged 10-19 years), female, of Korean nationality, showed smear-negative PTB or EPTB, had treatment outcomes reported by a public health centre, or were registered as TB-positive once. The rate of loss to follow-up was higher in patients who were male, foreign nationals, diagnosed with EPTB, or had their treatment outcome reported by institutions other than a public health centre. Mortality was higher among patients who were older ( $\geq 75$ years), male, of Korean nationality, diagnosed with smear-positive PTB, had their treatment outcome reported by the hospital, or were registered as TB-positive at least twice.

\section{Factors affecting treatment outcomes}

Table 4 shows the results of multivariate analysis of factors affecting treatment outcomes. Higher treatment success rates were seen in patients who were female or whose treatment outcome was reported by a public health centre. Conversely, lower treatment success rates were seen in patients who were registered in 2015 , were $\geq 45$ years old, were foreign nationals, were registered as TB-positive twice, or were classified as Q4 in the regional deprivation index.

Higher rates of loss to follow-up were seen in patients who were registered in 2015, were foreign nationals, were registered as TB-positive at least twice, or who were classified as Q4 or Q5 in the regional deprivation index. Female gender, smear-positive PTB, and treatment outcome reported by a public health centre were protective against loss to follow-up.

Higher mortality was associated with older age $(\geq$ 75 years), diagnosis of PTB (especially smear-positive PTB), treatment outcome reported by a hospital, and two registrations as TB-positive. On the other hand, lower mortality was associated with female gender, treatment outcome being was reported by a public health centre or clinic, and classification of Q5 in the regional deprivation index.

\section{Discussion}

To our knowledge, this is the first large-scale study to investigate the treatment outcomes, and factors affecting outcomes, of new patients with TB in Busan, South Korea. A major strength of our study was that it included a large cohort of TB patients, drawn from the population of a major city. Another strength is that we were able to include patients who transferred to other hospitals within the city, by using patient identification 
Table 2 Treatment outcomes of the study cohort by year

\begin{tabular}{lllll}
\hline & $\begin{array}{l}\text { Total } \\
(n=4732)\end{array}$ & $\begin{array}{l}2014 \\
(n=2455)\end{array}$ & $\begin{array}{l}2015 \\
(n=2277)\end{array}$ \\
\hline Cured & $955(20.2)$ & $603(24.6)$ & $352(15.5)$ & $p^{\text {a }}$ \\
Completed & $3015(63.7)$ & $1501(61.1)$ & $1514(66.5)$ & $<0.001$ \\
Failed & $4(0.1)$ & $3(0.1)$ & $1(0.0)$ & 0.001 \\
Lost to follow-up & $171(3.6)$ & $88(3.6)$ & $83(3.6)$ & 0.626 \\
Died & $379(8.0)$ & $192(7.8)$ & $187(8.2)$ & 0.911 \\
Not evaluated & $208(4.4)$ & $68(2.8)$ & $140(6.1)$ & 0.620 \\
\hline
\end{tabular}

Data are presented as numbers (\%)

${ }^{\mathrm{a} C}$ Comparison between 2014 and 2015

numbers to determine treatment outcomes. The Korean NTSS successfully registered $94.0 \%$ of all TB cases in 2014 [13], so it is likely that our results are representative of the overall TB status of Busan.

During the study period, the treatment success rate of new TB patients in Busan was $83.9 \%$, which was similar to that of South Korea as a whole $(84.4 \%$ for 2014 and $83.8 \%$ for 2015) [14]. However, neither rate met the 90\% target set by the WHO. This sub-optimal treatment success rate was mainly due to high mortality (8.0\%). One of the main risk factors for death of TB patients is old age. A lower in treatment success rate due to the high incidence of death of elderly TB patients is typical of both low- and high-TB incidence countries [15-17]. Several factors are associated with death in elderly TB patients. Besides aging per se, high mortality in elderly TB patients may be due to age-related comorbidities, reduced immune function, missed diagnosis and delayed treatment initiation due to atypical presentation of $\mathrm{TB}$, and difficulties in accessing healthcare services [18-21]. In our cohort, elderly TB patients ( $\geq 60$ years old) accounted for nearly $40 \%$ of all of the patients and, not surprisingly, old age was an independent risk factor for death. The number of elderly TB patients is expected to increase as life expectancy continues to improve, which presents major challenges to TB control in South Korea [22]. In addition to old age, male gender and PTB (especially smear-positive PTB) were also independently associated with high mortality in our study, which is consistent with previous reports [17, 23]. Higher death rates in male patients may be due to behaviours such as alcohol consumption, smoking, and non-adherence to treatment [24-27]. Patients with smear-positive PTB may have advanced disease. Treatment outcomes being reported by a hospital was another risk factor for death in our study, but this could be due to selection bias: a considerable number of TB patients who have chronic critical illness and limited options for treatment are transferred to such hospitals in the post-infectious stage, likely leading to higher acuity of hospital patients. Unexpectedly, Q5 of the regional deprivation index was associated with lower mortality. However, this was attributable to the higher rate of loss to follow-up in the Q5 group; we estimate that a significant proportion of the patients lost to follow-up in the Q5 group were indeed dead.

To reduce death from TB, interventions for elderly patients should be prioritised. Elderly patients are more likely to show an atypical presentation of TB, so actively searching for cases would facilitate early diagnosis and timely treatment, which would in turn likely reduce mortality $[28,29]$. The standard sputum AFB smear has low sensitivity in elderly patients, making TB detection more difficult, but molecular tests such as the Xpert MTB/RIF assay could overcome this problem [30]. Administrative support, such as financial support and help with transportation, could improve access to healthcare services for vulnerable elderly patients. TB-related deaths are common in the intensive treatment phase [7, 16], so intensive monitoring early in this treatment period should be considered not only for elderly patients, but also for patients with other risk factors for death (e.g. male, smear-positive PTB, non-adherence to treatment).

The number of elderly TB patients is expected to increase in the future, and efforts to reduce mortality of elderly patients may be stymied by the inherent demographic characteristics of this population. In addition, all new patients with TB had low rates of treatment failure which would be difficult to decrease further. Therefore, to achieve WHO targets, a paradigm shift in TB control strategies may be necessary to reduce non-adherence to treatment among patients in Busan. Non-adherence results in serious problems not only for the patients themselves (including development of complications, acquired drug resistance, and death), but also for communities in terms of the spread of infection [31]. In our study, male gender, foreign nationality, smear-negative PTB and EPTB, and treatment outcome reported by institutions other than public health centres were risk factors for loss to follow-up, which was consistent with previous studies [32-35]. The number of TB patients of foreign nationality 
Table 3 Treatment outcomes of the study cohort according to baseline characteristics

\begin{tabular}{|c|c|c|c|c|c|c|c|}
\hline & $\begin{array}{l}\text { Total }^{a} \\
(n=4732)\end{array}$ & $\begin{array}{l}\text { Success } \\
(n=3970)\end{array}$ & $P$ & $\begin{array}{l}\text { Lost to follow-up } \\
(n=379)\end{array}$ & $P$ & $\begin{array}{l}\text { Died } \\
(n=379)\end{array}$ & $P$ \\
\hline Age, years & & & $<0.001$ & & 0.229 & & $<0.001$ \\
\hline $0-9$ & 11 & $10(90.9)$ & & $0(0.0)$ & & $1(9.1)$ & \\
\hline 10-19 & 196 & $187(95.4)$ & & $9(4.6)$ & & $0(0.0)$ & \\
\hline $20-44$ & 1451 & $1331(91.7)$ & & $107(7.4)$ & & $10(0.7)$ & \\
\hline $45-59$ & 1190 & $1042(87.6)$ & & $106(8.9)$ & & $41(3.5)$ & \\
\hline $60-74$ & 1117 & $893(80.0)$ & & $97(8.7)$ & & $127(11.4)$ & \\
\hline$\geq 75$ & 767 & $507(66.1)$ & & $60(7.8)$ & & $200(26.1)$ & \\
\hline Sex & & & $<0.001$ & & $<0.001$ & & 0.002 \\
\hline Male & 2765 & $2249(81.3)$ & & $264(9.5)$ & & $250(9.0)$ & \\
\hline Female & 1967 & $1721(87.5)$ & & $115(5.8)$ & & $129(6.6)$ & \\
\hline Nationality & & & $<0.001$ & & $<0.001$ & & 0.009 \\
\hline Korean & 4654 & 3919 (84.2) & & $352(7.6)$ & & $379(8.1)$ & \\
\hline Foreign & 78 & $51(65.4)$ & & 27 (34.6) & & $0(0.0)$ & \\
\hline Type of tuberculosis & & & 0.011 & & 0.018 & & $<0.001$ \\
\hline Pulmonary, smear positive & 1464 & $1193(81.5)$ & & $98(6.7)$ & & $172(11.7)$ & \\
\hline Pulmonary, smear negative & 2307 & $1961(85.0)$ & & $186(8.1)$ & & $158(6.8)$ & \\
\hline Extrapulmonary & 961 & $816(84.9)$ & & $95(9.9)$ & & $49(5.1)$ & \\
\hline Type of institution ${ }^{c}$ & & & $<0.001$ & & 0.043 & & $<0.001$ \\
\hline Public health centre & 551 & $519(94.2)$ & & $27(4.9)$ & & $4(0.7)$ & \\
\hline General hospital $^{d}$ & 3489 & $2910(83.4)$ & & $298(8.5)$ & & $278(8.0)$ & \\
\hline PPM participating & 2542 & $2117(83.3)$ & & $212(8.3)$ & & $210(8.3)$ & \\
\hline PPM non-participating & 947 & $793(83.7)$ & & $86(9.1)$ & & $68(7.2)$ & \\
\hline Hospital & 545 & $409(75.0)$ & & $40(7.3)$ & & $96(17.6)$ & \\
\hline Clinic & 147 & $132(89.8)$ & & $14(9.5)$ & & $1(0.7)$ & \\
\hline Number of registrations as TB-positive & & & $<0.001$ & & 0.148 & & $<0.001$ \\
\hline 1 & 3711 & $3169(85.4)$ & & $284(7.7)$ & & $254(6.8)$ & \\
\hline 2 & 849 & $669(78.8)$ & & $76(9.0)$ & & $104(12.2)$ & \\
\hline$\geq 3$ & 172 & $132(76.7)$ & & 19 (11.0) & & $21(12.2)$ & \\
\hline Regional deprivation index (quintile) $^{\mathrm{e}}$ & & & 0.556 & & 0.820 & & 0.201 \\
\hline Q1 & 1135 & $85.4 \pm 5.6$ & & $3.1 \pm 2.8$ & & $8.0 \pm 3.7$ & \\
\hline Q2 & 1237 & $83.7 \pm 6.1$ & & $2.9 \pm 2.0$ & & $8.3 \pm 5.5$ & \\
\hline Q3 & 1213 & $83.1 \pm 6.5$ & & $3.9 \pm 3.1$ & & $8.6 \pm 5.6$ & \\
\hline Q4 & 729 & $80.7 \pm 12.4$ & & $3.7 \pm 4.0$ & & $10.6 \pm 8.8$ & \\
\hline Q5 & 344 & $83.8 \pm 11.6$ & & $4.1 \pm 6.5$ & & $5.5 \pm 6.0$ & \\
\hline
\end{tabular}

PPM public-private mix, TB tuberculosis

Data are presented as numbers (\%) or means \pm standard deviations

${ }^{a}$ Including four patients whose treatment failed

${ }^{b}$ Including 208 patients whose treatment outcome was not evaluated

'Type of institution that reported the final treatment outcome

${ }^{\mathrm{d}}$ There was no significant difference between PPM participating and non-participating general hospitals in rates of treatment success, loss to follow-up, or death

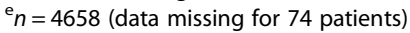

has increased in South Korea (from 849 patients in 2010 to 2045 in 2017) [2]. The higher rate of loss to follow-up in these foreign national populations may be due to difficulties in accessing healthcare services due to legal, employment, economic, or language problems [33]. The higher rate of loss to follow-up in cases of EPTB may be due to excessively long treatment durations, lack of motivation due to lower symptom severity compared to PTB, or reduced attention from $\mathrm{TB}$ control programs due to lower infectivity [36, 37]. 
Table 4 Multivariate analysis of factors affecting treatment outcomes

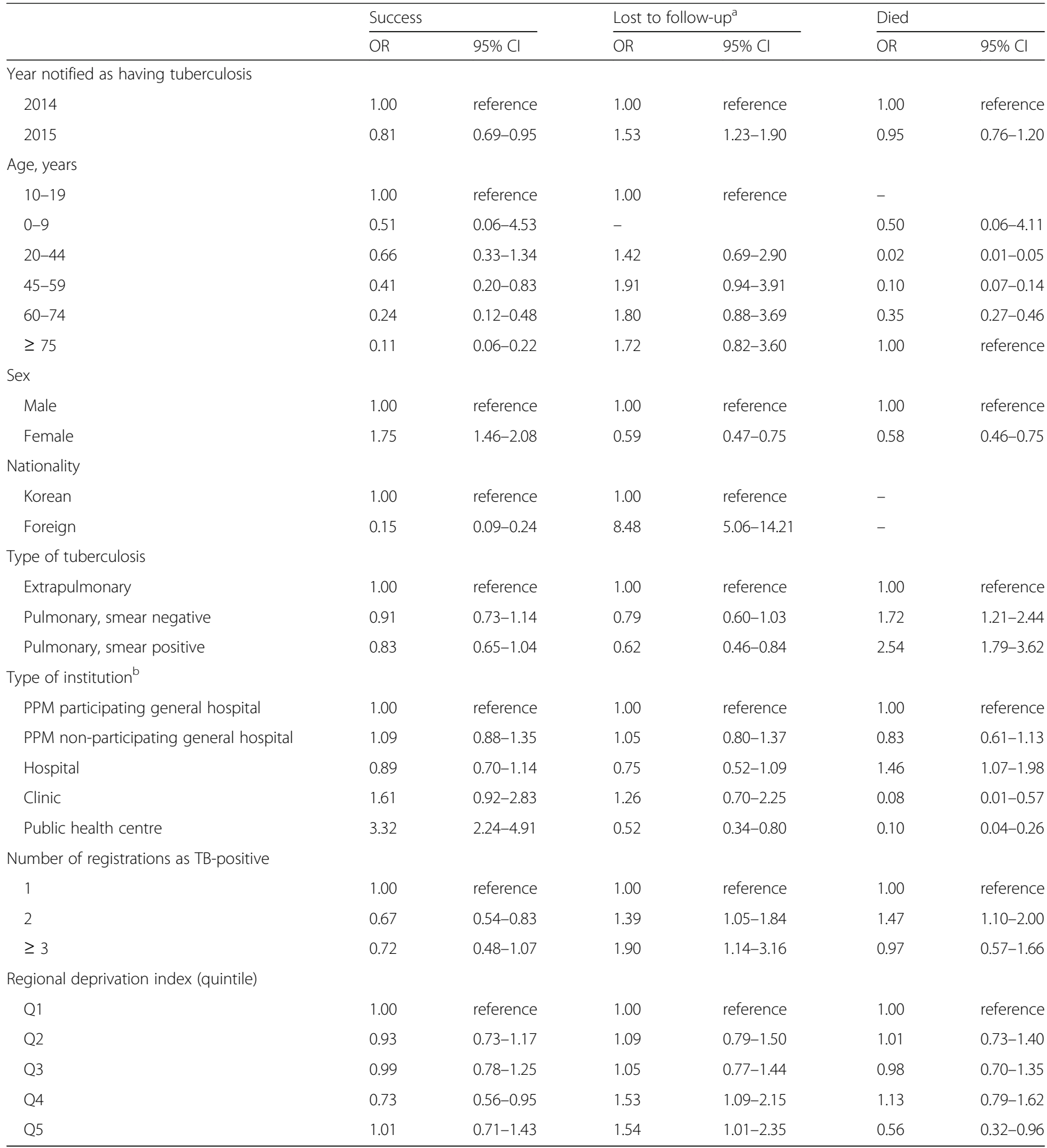

Cl confidence interval, $O R$ odds ratio, $P P M$ public-private mix, $T B$ tuberculosis

ancluding 208 patients whose treatment outcome was not evaluated

${ }^{\mathrm{b}}$ Type of institution that reported final treatment outcome

To improve patient compliance, PPM collaborations should be expanded throughout South Korea, as well as in Busan specifically, to enhance TB control in the private sector. A previous South Korean study showed that PPM collaborations decreased the rate of loss to follow-up in the private sector [38]. For non-adherent patients of foreign nationality, international collaborations to ensure continuity of treatment after departure could be useful, in addition to administrative support to improve access to healthcare services [39]. Patient- 
centred care is also important for reducing non-adherence to treatment. This may include providing sufficient information on TB to patients, improving communication between patients and healthcare providers, and providing social support to help overcome the stigma of TB. Although studies of the efficacy of directly observed therapy (DOT) have reported inconclusive results, DOT may be considered for non-compliant patients $[3,35,40$, 41].

There were several limitations to this study. First, socioeconomic parameters that could affect patient treatment outcomes were not recorded (e.g. smoking status, alcohol consumption, level of education, degree of employment, and economic status). We used a regional deprivation index as an indirect indicator of socioeconomic status, but it showed inconsistent associations with treatment outcomes. Because the regional deprivation index is based on the data of a given area (town level), not a patient, it might not reflect the socioeconomic status of the individual patient. Also, Busan is one of the most highly developed metropolitan cities in South Korea, so differences in regional deprivation might not be particularly significant. Further, socioeconomic differences may be diluted by free-of-charge hospital visits for TB and relatively easy access to healthcare services for all patients in Busan. Second, we did not include previously treated patients, or those with RR- or MDR-TB in the analyses. These patients are well known as high-risk groups for poor treatment outcomes. Further investigations are needed on these vulnerable populations. Finally, we could not distinguish between TB-related and non-TB related deaths. Although the WHO defines TB death as that occurring during TB treatment, irrespective of cause, knowing the actual cause of death may help to improve TB control programs.

\section{Conclusion}

Treatment outcomes of new TB patients were found to be sub-optimal in Busan, South Korea. To reduce mortality and loss to follow-up rates, and therefor increase the treatment success rate, TB control programs should involve close monitoring and provide more socioeconomic support to patients at high risk for poor treatment outcomes.

\section{Abbreviations}

AFB: Acid-fast bacilli; DOT: Directly observed therapy; EPTB: Extrapulmonary tuberculosis; KCDC: Korea Centers for Disease Control and Prevention; MDR: Multidrug-resistant; NTP: National tuberculosis program; NTSS: National TB Surveillance System; PPM: Public-private mix; PTB: Pulmonary tuberculosis; RR: Rifampin-resistant; TB: Tuberculosis; WHO: World Health Organization
Funding

None.

\section{Availability of data and materials}

The datasets analysed in the current study are available from the corresponding author on reasonable request.

\section{Authors' contributions}

JM, CK and HS conceived the initial idea and the design of the study. All authors contributed to data analyses, and interpreted the results. JM and HS drafted the manuscript. All authors revised the manuscript and approved the final version.

\section{Ethics approval and consent to participate}

The study protocol was reviewed and approved by the Institutional Review Board of Pusan National University Hospital (IRB approval number, C-1805001-066). The requirement for informed consent was waived by the Institutional Review Board of Pusan National University Hospital because this was a retrospective study of routinely collected monitoring data. The study dataset was private and was provided by the Department of Health and Hygiene of Busan City. Administrative permission for review and analysis of patient records was obtained from the Department of Health and Hygiene of Busan City. The data were de-identified prior to being analysed.

\section{Consent for publication}

Not applicable.

\section{Competing interests}

The authors declare that they have no competing interests.

\section{Publisher's Note}

Springer Nature remains neutral with regard to jurisdictional claims in published maps and institutional affiliations.

\section{Author details}

${ }^{1}$ Department of Internal Medicine, College of Medicine, Pusan National University, Busan, South Korea. ${ }^{2}$ Medical Research Institute, Pusan National University Hospital, Busan, South Korea. ${ }^{3}$ Busan Center for Infectious Disease Control and Prevention, Pusan National University Hospital, 179 Gudeok-ro,

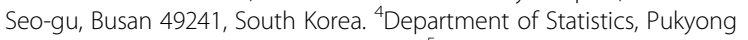
National University, Busan, South Korea. ${ }^{5}$ Department of Preventive Medicine, College of Medicine, Pusan National University, Busan, South Korea.

Received: 31 May 2018 Accepted: 30 November 2018

Published online: 13 December 2018

\section{References}

1. World Health Organization. Global tuberculosis report, 2017. Geneva: WHO; 2017. WHO/HTM/TB/2017.23

2. Korea Centers for Disease Control and Prevention. Annual Report on the Notified Tuberculosis Patients in Korea: 2017. http://tbzero.cdc.go.kr/tbzero/ main.do. Accessed 1 Mar 2018.

3. Kim JH, Yim JJ. Achievements in and challenges of tuberculosis control in South Korea. Emerg Infect Dis. 2015;21:1913-20.

4. World Health Organization. The END TB strategy. Geneva: WHO; 2015. WHO/ HTM/TB/2015.19

5. World Health Organization. Definitions and reporting framework for tuberculosis-2013 revision. Geneva: WHO; 2013. WHO/HTM/TB/2013.2

6. Choi H, Lee M, Chen RY, Kim Y, Yoon S, Joh JS, et al. Predictors of pulmonary tuberculosis treatment outcomes in South Korea: a prospective cohort study, 2005-2012. BMC Infect Dis. 2014;14:360.

7. Kwon YS, Kim YH, Song JU, Jeon K, Song J, Ryu YJ, et al. Risk factors for death during pulmonary tuberculosis treatment in Korea: a multicenter retrospective cohort study. J Korean Med Sci. 2014;29:1226-31.

8. Choi H, Chung H, Muntaner C, Lee M, Kim Y, Barry CE, et al. The impact of social conditions on patient adherence to pulmonary tuberculosis treatment. Int J Tuberc Lung Dis. 2016;20:948-54.

9. Korea Centers for Disease Control and Prevention. Annual Report on the Notified Tuberculosis Patients in Korea: 2015. http://tbzero.cdc.go.kr/tbzero/ main.do. Accessed 1 Mar 2016. 
10. World Health Organization. Global tuberculosis report, 2016. Geneva: WHO 2016. WHO/HTM/TB/2016.13

11. Joint Committee for the Revision of Korean Guidelines for Tuberculosis, Korean Centers for Disease Control and Prevention. Korean guidelines for tuberculosis. 2nd ed; 2014. http://www.lungkorea.org/bbs/index.html?code= guide\&page $=2$. Accessed 1 Aug 2014

12. Korea Institute for Health and Affairs. Developing health inequalities indicators and monitoring the status of health inequalities in Korea. http:// www.ndsl.kr/ndsl/search/detail/report/reportSearchResultDetail.do?cn= TRKO201400012926. Accessed 1 Nov 2013.

13. Kang HY, Yoo H, Park W, Go U, Jeong E, Jung KS, et al. Tuberculosis notification completeness and timeliness in the Republic of Korea during 2012-2014. Osong Public Health Res Perspect. 2016;7:320-6.

14. Korea Centers for Disease Control and Prevention. Report on the tuberculosis control. 2015-4. http://tbzero.cdc.go.kr/tbzero/main.do. Accessed 2 Jun 2015.

15. Waitt CJ, Squire SB. A systematic review of risk factors for death in adults during and after tuberculosis treatment. Int J Tuberc Lung Dis. 2011;15:871-85.

16. Chiang CY, Lee JJ, Yu MC, Enarson DA, Lin TP, Luh KT. Tuberculosis outcomes in Taipei: factors associated with treatment interruption for 2 months and death. Int J Tuberc Lung Dis. 2009;13:105-11.

17. Karo B, Hauer B, Hollo V, van der Werf MJ, Fiebig L, Haas W. Tuberculosis treatment outcome in the European Union and European economic area: an analysis of surveillance data from 2002-2011. Euro Surveill. 2015;20(49).

18. Zevallos M, Justman JE. Tuberculosis in the elderly. Clin Geriatr Med. 2003; 19:121-38.

19. Vasankari T, Holmström P, Ollgren J, Liippo K, Kokki M, Ruutu P. Risk factors for poor tuberculosis treatment outcome in Finland: a cohort study. BMC Public Health. 2007;7:291.

20. Farah MG, Tverdal A, Steen TW, Heldal E, Brantsaeter AB, Bjune G. Treatment outcome of new culture positive pulmonary tuberculosis in Norway. BMC Public Health. 2005;5:14.

21. Negin J, Abimbola S, Marais BJ. Tuberculosis among older adults-time to take notice. Int J Infect Dis. 2015;32:135-7.

22. Kwon YS, Chi SY, Oh IJ, Kim KS, Kim YI, Lim SC, et al. Clinical characteristics and treatment outcomes of tuberculosis in the elderly: a case control study. BMC Infect Dis. 2013:13:121.

23. Lefebvre N, Falzon D. Risk factors for death among tuberculosis cases: analysis of European surveillance data. Eur Respir J. 2008;31:1256-60.

24. Valin N, Hejblum G, Borget I, Mallet HP, Antoun F, Che D, et al. Management and treatment outcomes of tuberculous patients, eastern Paris, France, 2004. Int J Tuberc Lung Dis. 2009;13:881-7.

25. Diel R, Niemann S. Outcome of tuberculosis treatment in Hamburg: a survey, 1997-2001. Int J Tuberc Lung Dis. 2003;7:124-31.

26. Caylà JA, Caminero JA, Rey R, Lara N, Vallés X, Galdós-Tangüis H. Working group on completion of tuberculosis treatment in Spain. Current status of treatment completion and fatality among tuberculosis patients in Spain. Int J Tuberc Lung Dis. 2004;8:458-64.

27. Balasubramanian R, Garg R, Santha T, Gopi PG, Subramani R, Chandrasekaran V, et al. Gender disparities in tuberculosis: report from a rural DOTS programme in South India. Int J Tuberc Lung Dis. 2004;8:323-32.

28. Hauer B, Brodhun B, Altmann D, Fiebig L, Loddenkemper R, Haas W. Tuberculosis in the elderly in Germany. Eur Respir J. 2011;38:467-70.

29. Velayutham BR, Nair D, Chandrasekaran V, Raman B, Sekar G, Watson B, et al. Profile and response to anti-tuberculosis treatment among elderly tuberculosis patients treated under the TB control programme in South India. PLoS One. 2014;9:e88045.

30. Pantoja A, Fitzpatrick C, Vassall A, Weyer K, Floyd K. Xpert MTB/RIF for diagnosis of tuberculosis and drug-resistant tuberculosis: a cost and affordability analysis. Eur Respir J. 2013;42:708-20.

31. Kigozi G, Heunis C, Chikobvu P, Botha S, van Rensburg D. Factors influencing treatment default among tuberculosis patients in a high burden province of South Africa. Int J Infect Dis. 2017;54:95-102.

32. Kapella BK, Anuwatnonthakate A, Komsakorn S, Moolphate S, Charusuntonsri $\mathrm{P}$, Limsomboon $\mathrm{P}$, et al. Directly observed treatment is associated with reduced default among foreign tuberculosis patients in Thailand. Int J Tuberc Lung Dis. 2009;13:232-7.

33. Min GH, Kim Y, Lee JS, Oh JY, Hur GY, Lee YS, et al. Social and clinical characteristics of immigrants with tuberculosis in South Korea. Yonsei Med J. 2017:58:592-7.
34. Muture BN, Keraka MN, Kimuu PK, Kabiru EW, Ombeka VO, Oguya F. Factors associated with default from treatment among tuberculosis patients in Nairobi province, Kenya: a case control study. BMC Public Health. 2011;11:696.

35. Bhavnani D, Lancki N, Winter I, Macaraig M. Treatment outcomes of patients with tuberculosis in new York City. J Public Health Manag Pract. 2015;21:E11-8.

36. Tattevin P, Chapplain JM, Lesprit P, Billy C, Roblot F, Alfandari S, et al. Tuberculosis treatment duration in France: from guidelines to daily practice. Eur J Intern Med. 2006;17:427-9.

37. Santha T, Garg R, Frieden TR, Chandrasekaran V, Subramani R, Gopi PG, et al. Risk factors associated with default, failure and death among tuberculosis patients treated in a DOTS programme in Tiruvallur District, South India, 2000. Int J Tuberc Lung Dis. 2002;6:780-8.

38. Kim HJ, Bai GH, Kang MK, Kim SJ, Lee JK, Cho SI, et al. A public-private collaboration model for treatment intervention to improve outcomes in patients with tuberculosis in the private sector. Tuberc Respir Dis. 2009; 66:349-57.

39. Millett ER, Noel D, Mangtani P, Abubakar I, Kruijshaar ME. Factors associated with being lost to follow-up before completing tuberculosis treatment: analysis of surveillance data. Epidemiol Infect. 2013;141:1223-31.

40. Karumbi J, Garner P. Directly observed therapy for treating tuberculosis. Cochrane Database Syst Rev. 2015;29:CD003343.

41. Pasipanodya JG, Gumbo T. A meta-analysis of self-administered vs directly observed therapy effect on microbiologic failure, relapse, and acquired drug resistance in tuberculosis patients. Clin Infect Dis. 2013;57:21-31.

\section{Ready to submit your research? Choose BMC and benefit from:}

- fast, convenient online submission

- thorough peer review by experienced researchers in your field

- rapid publication on acceptance

- support for research data, including large and complex data types

- gold Open Access which fosters wider collaboration and increased citations

- maximum visibility for your research: over 100M website views per year

At BMC, research is always in progress.

Learn more biomedcentral.com/submissions 